\title{
TRABALHO NOTURNO E MORBIDADES DE TRABALHADORES DE ENFERMAGEM
}

\author{
Divanise de Carvalho Dias Maynardes ${ }^{1}$, Leila Maria Mansano Sarquis² ${ }^{2}$ Ana Lúcia Cardoso Kirchhof ${ }^{3}$
}

\begin{abstract}
RESUMO: Pesquisa exploratória descritiva realizada em um hospital público de Curitiba. A população foi composta por 173 trabalhadores de enfermagem do turno noturno, com objetivo de identificar os principais agravos à saúde desses trabalhadores. Responderam ao questionário $66,5 \%$ dos trabalhadores, sendo $84 \%$ do sexo feminino. O processo de morbidade expresso pelos trabalhadores do noturno comprova o desgaste no processo saúde e doença. Os sintomas mais expressivos foram varizes, fadiga, cervicodorsolombalgia e irritabilidade acima de $50 \%$. Os sintomas registrados após o descanso confirmam que o trabalhador ainda sente-se cansado e desanimado e permanece ainda com sono excessivo, o que confirma que o trabalhador retorna ao trabalho em situação de fadiga. As consequências acarretadas à saúde são vistas como um grande problema e medidas de intervenção devem ser estudadas para intervir no processo saúde doença dos trabalhadores de enfermagem.
\end{abstract}

PALAVRAS-CHAVE: Serviço hospitalar de enfermagem; Saúde do trabalhador; Trabalho noturno; Recursos humanos em saúde.

\section{NOCTURNE JOB AND NURSING WORKERS MORBIDITY}

\begin{abstract}
Descriptive exploratory research in a Public University in Curitiba. The population was composed of 173 nursing workers of the night shift. The goal was to identify the main health problems of workers of the night nursing shift. The questionnaire was answered by $66,5 \%$ workers, being $84 \%$ of them females. The process of morbidity expressed by employees of the night shifts shows the wear process in health and disease. The most significant ones were varicose veins, fatigue, irritability and cervical injuries above $50 \%$. The symptoms recorded after the work rest confirm that the employee still feels tired and discouraged and remain excessively sleepy, confirming that the employee returns to work in a state of fatigue. The consequences entailing health are seen as a major problem and the intervention measures should be considered to intervene in the health disease process of nursing workers.
\end{abstract}

KEYWORDS: Hospital nursing service; Occupational health; Night work; Health human resources.

\section{TRABAJO NOCTURNO Y MORBIDAD DE TRABAJADORES DE ENFERMERÍA}

RESUMEN: Investigación exploratorio-descriptiva realizada en un Hospital Público de Curitiba. La población fue compuesta por 173 trabajadores de enfermería del turno nocturno, con objetivo de identificar los principales agravantes de salud de estos trabajadores. Respondieron al cuestionario $66,5 \%$ de los trabajadores, siendo $84 \%$ del sexo femenino. El proceso de morbilidad expresado por los empleados de la noche comprueba el desgaste en el proceso salud y enfermedad. Los síntomas más significativos fueron varices, fatiga, irritabilidad y cervicodorsolombalgias e irritabilidad encima del 50\%. Los síntomas registrados después del descanso confirman que el empleado todavía se siente cansado y desanimado y permanece aún con sueño excesivo, lo que confirma que el trabajador vuelve del trabajo en situación de fatiga. Las consecuencias acarreadas a la salud son vistas como un problema importante y medidas de intervención deben ser estudiadas para intervenir en el proceso salud enfermedad de los trabajadores de enfermería.

PALABRAS CLAVE: Servicio hospitalario de enfermería; Salud del trabajador; Trabajo nocturno; Recursos humanos en salud.

\footnotetext{
${ }^{1}$ Enfermeira. Membro do Grupo de Estudo Multidisciplinar de Saúde do Adulto-GEMSA, da Universidade Federal do Paraná-UFPR. ${ }^{2}$ Enfermeira. Professor Adjunto do Curso de Graduação de Enfermagem da UFPR. Membro do GEMSA. Membro do Grupo de Pesquisa de Qualidade de Vida da Escola de Enfermagem da Universidade de São Paulo-EEUSP.

${ }^{3}$ Enfermeira. Professora aposentada do Departamento de Enfermagem da Universidade Federal de Santa Catarina-UFSC. Membro do Grupo de Estudos e Pesquisas em Administração e Gerência do Cuidado em Enfermagem e Saúde-GEPADES-UFSC.
}

Autor correspondente:

Leila Maria Mansano Sarquis

Universidade Federal do Paraná

Rua Padre Camargo, 120 - 80060-240 - Curitiba-PR, Brasil

Recebido: 10/06/09

E-mail: leila.sarquis@ufpr.br

Aprovado: 03/11/09 


\section{INTRODUÇÃO}

A política oficial da Saúde do Trabalhador ${ }^{(1)}$ tem como objetivo a promoção e a proteção da saúde dos trabalhadores, pelo desenvolvimento de ações de vigilância dos riscos e condições ambientais de trabalho e dos agravos à saúde, promovendo a prestação da assistência, tratamento e reabilitação de forma integrada por meio de uma atuação multiprofissional, interdisciplinar e intersetorial.

A Saúde no Trabalho, de acordo com a Organização Mundial de Saúde-OMS vem garantir o estado de saúde integral ao trabalhador, com o objetivo de prolongar a expectativa de vida e minimizar a incidência de incapacidade, de doença, de dor e desconforto, incluindo a preservação da capacidade de reserva e dos mecanismos de adaptação, a provisão de realização pessoal, tornando-os sujeitos criativos $^{(2)}$.

As longas jornadas de trabalho, realizadas pelos trabalhadores de saúde são fonte de desgaste, submetendo estes trabalhadores à diminuição do seu período de descanso e lazer devido à necessidade de possuírem mais de um vínculo empregatício(3).

A enfermagem é uma das profissões da área de saúde cuja essência e especificidade é o cuidado ao ser humano, pelo desenvolvimento de atividades em equipe que proporcionam a promoção, recuperação e reabilitação da saúde e a prevenção de doenças, atuando no indivíduo, na família e na comunidade ${ }^{(4)}$. Como as atividades de enfermagem nos hospitais são ininterruptas, os profissionais são organizados em turnos de trabalho. Para o trabalhador de enfermagem o turno de 12 horas de trabalho diário (diurno ou noturno), seguido de 36 horas de descanso, já é tradição no Brasil ${ }^{(4)}$.

Os ritmos biológicos associados ao claro e escuro são conhecidos como ritmos circadianos e de acordo com a espécie, o período pode variar de 20 a 28 horas. O período de oscilação dos ritmos circadianos nos seres humanos possui uma frequência próxima das 24 horas $^{(5)}$.

Através do ritmo circadiano há uma sincronização interna que vem em resposta ao meio externo, a qual ocorre uma variedade de medidas fisiológicas e psicológicas: o sono, a secreção hormonal, a temperatura corporal, a excreção urinária, o alerta subjetivo, o humor e o desempenho, produzindo valores máximos e mínimos quase sempre no mesmo horário ao longo das 24 horas do $\operatorname{dia}^{(5-6)}$.

Os seres humanos possuem uma ritmicidade natural para muitas funções corporais, dentre elas as fisiológicas, as psicológicas e as comportamentais. $\mathrm{O}$ trabalhador noturno, por inverter o ciclo sono-vigília, dormir durante o dia e trabalhar durante a noite induz a uma dessincronização dos ritmos biológicos e cicardianos $^{(7)}$.

A desordem da estrutura dos ritmos circadianos causa mal-estar, fadiga, sonolência, insônia, irritabilidade, prejuízo da agilidade mental e do desempenho e, consequentemente, diminuição da eficiência. O sono diurno é prejudicado pelas condições ambientais não favoráveis como iluminação, ruídos e acontecimentos domésticos, modificando a distribuição das fases do sono e interferindo em sua propriedade restauradora. A dessincronização dos ritmos circadianos a longo prazo pode levar a distúrbios severos e persistentes do sono, como fadiga crônica e síndrome psiconeurótica (ansiedade e depressão crônica), requerendo tratamento com drogas psicotrópicas ou hipnóticas( ${ }^{(8)}$.

O hospital, tradicional local de trabalho da enfermagem, se caracteriza por ser fonte de cargas de trabalho ${ }^{(9)}$ físicas, biológicas, químicas, implicando em insalubridade e periculosidade que se não forem devidamente controladas implicam em acidentes e doenças. Dentro deste contexto procurou-se identificar os principais agravos à saúde dos trabalhadores de enfermagem do período noturno, segundo seus relatos e tendo como questão norteadora: quais são as morbidades relatadas pelos trabalhadores de enfermagem do período noturno?

\section{METODOLOGIA}

Foi realizada pesquisa exploratória-descritiva em um Hospital Universitário de Curitiba de grande porte, centro de referência na atenção à saúde de média e alta complexidade do Sistema Único de Saúde-SUS. A população da pesquisa foi composta pelos trabalhadores de enfermagem, tendo como critério de inclusão ser enfermeiro, auxiliar ou técnico de enfermagem do período noturno, totalizando 173trabalhadores de enfermagem.

A coleta de dados foi realizada no período de fevereiro a março de 2008, por meio da aplicação de um questionário com perguntas abertas e fechadas, abordando os sinais e sintomas que acometem esses trabalhadores conforme descritos em literatura ${ }^{(4-10)}$

$\mathrm{Na}$ análise dos dados utilizou-se a estatística descritiva, compondo as maiores frequências

Cogitare Enferm 2009 Out/Dez; 14(4):703-8 
encontradas. Os dados foram descritos e sistematizados em planilhas e inseridos num banco de dados no programa Microsoft Office Excel 2003. As informações fornecidas pelos trabalhadores de enfermagem foram processadas e categorizadas de acordo com as variáveis definidas pelo instrumento de coleta dos dados e apresentadas na forma de tabelas. Com base na revisão de literatura previamente feita, buscou-se identificar e discutir os principais agravos que acometem o trabalhador do período noturno.

O estudo obteve parecer favorável pelo Comitê e Ética em Pesquisa do Setor de Ciências da Saúde sob protocolo n. 462.142.07.12; CAAE 0269.0.208.091-07.

Participaram da pesquisa 173 trabalhadores: 18 enfermeiros, 32 técnicos de enfermagem, 118 auxiliares de enfermagem e 5 não responderam a que ocupação pertenciam. Esses trabalhadores assinaram o Termo de Consentimento Livre e Esclarecido, sendo garantidos os princípios da Resolução 196/96 do Ministério da Saúde.

\section{RESULTADOS}

Os resultados estão apresentados a partir da caracterização e perfil dos trabalhadores de enfermagem do período noturno e logo após os processos de morbidades e a qualidade de sono relatado por eles.

A maioria dos profissionais de enfermagem pesquisados são casados $(50,29 \%)$ e estão na faixa etária de 41 a 50 anos (34,10\%); 74\% dos trabalhadores entrevistados possuem filhos. Além disso, confirma-se a constatação da predominância do sexo feminino (84\%), o que vem confirmar essa categoria como eminentemente feminina.

Percebe-se na Tabela 1 a grande quantidade de sintomas/doenças relatados pelos trabalhadores, cujas manifestações ocorrem tanto na sua integridade física quanto psíquica. Eles também relataram em menor proporção outros problemas, como ganho de peso, dores nos membros inferiores e irritação ocular.

No questionário aplicado os trabalhadores, na opção "necessidade de trabalhar a noite", responderam com maior frequência "possuir outro emprego (45,66\%)”. No entanto devem-se destacar também as opções "estudo (16,76\%)", "filhos (13,87\%)" e "salário (10,98\%)", pois as mesmas mostram os outros compromissos em que se envolvem, para além do trabalho.
Tabela 1 - Caracterização dos trabalhadores segundo a idade, estado civil, tempo de trabalho no período noturno e sintomas e doenças relatados pelos profissionais de enfermagem noturno. Curitiba, 2008

\begin{tabular}{lcc}
\hline Variáveis & Trabalhadores* & $\%$ \\
\hline Idade (anos) & 4 & 2,31 \\
18 a 25 & 20 & 11,56 \\
26 a 30 & 46 & 26,59 \\
31 a 40 & 59 & 34,10 \\
41 a 50 & 22 & 12,72 \\
$>\quad 50$ & 22 & 12,72 \\
Não responderam & & \\
Estado Civil & 87 & 50,29 \\
Casado & 36 & 20,81 \\
Solteiro & 22 & 12,72 \\
Separado & 5 & 2,89 \\
Viúvo & 11 & 6,36 \\
Outros & 12 & 6,93 \\
Não responderam & &
\end{tabular}

Necessidade de trabalhar à noite*

$\begin{array}{lcc}\text { Outro emprego } & 79 & 45,66 \\ \text { Estudo } & 29 & 16,76 \\ \text { Filhos } & 24 & 13,87 \\ \text { Salário } & 19 & 10,98 \\ \text { Opção própria } & 15 & 8,67 \\ \text { Distância casa-trabalho } & 3 & 1,73\end{array}$

Tempo de trabalho no período noturno (horas)

$\begin{array}{lcc}\text { Até } 5 & 44 & 25,43 \\ 5 \text { a } 10 & 40 & 23,13 \\ 10 \text { a } 15 & 30 & 17,34 \\ 15 \text { a } 20 & 26 & 15,02 \\ 20 \text { a } 25 & 14 & 8,09 \\ \text { Mais de 25 } & 7 & 4,05 \\ \text { Não responderam } & 12 & 6,94 \\ \text { Sintomas/Doenças* } & & \\ \text { Varizes de MMII } & 99 & 57,23 \\ \text { Fadiga/perda de energia } & 95 & 54,21 \\ \text { Cervicodorsolombalgia } & 95 & 54,21 \\ \text { Irritabilidade } & 90 & 52,02\end{array}$


Continuação

\begin{tabular}{|c|c|c|}
\hline Variáveis & Trabalhadores* & $\%$ \\
\hline $\begin{array}{l}\text { Dificultade em se } \\
\text { concentrar/pensar }\end{array}$ & 70 & 40,46 \\
\hline Enxaquecas & 66 & 38,15 \\
\hline Dor de estômago & 65 & 37,57 \\
\hline Sono excessivo & 63 & 36,42 \\
\hline Insônia & 63 & 36,42 \\
\hline Azia & 58 & 33,53 \\
\hline Gastrite & 53 & 30,63 \\
\hline Discutir com facilidade & 48 & 27,75 \\
\hline Constipação & 44 & 25,44 \\
\hline Hipertensão arterial & 41 & 23,70 \\
\hline Sentimento de desesperança & 38 & 21,96 \\
\hline $\begin{array}{l}\text { Infecções de garganta } \\
\text { repetidas }\end{array}$ & 32 & 18,50 \\
\hline Dermatites de contato & 31 & 17,92 \\
\hline Problemas respiratórios & 28 & 10,40 \\
\hline Urticária & 18 & 8,67 \\
\hline Pensamentos de morte & 15 & 8,09 \\
\hline Candidíase & 14 & 8,09 \\
\hline Diabetes miellitus & 11 & 6,36 \\
\hline Diarreia & 10 & 5,78 \\
\hline Doenças cardíacas & 9 & 5,20 \\
\hline Perda de peso & 7 & 4,05 \\
\hline Total & 173 & 100,00 \\
\hline
\end{tabular}

*Trabalhadores realizaram múltipla escolha, não fechando o total de trabalhadores e a porcentagem

Na pergunta aberta do questionário "Está em tratamento de alguma doença?”, os trabalhadores relataram apresentar os seguintes agravos: hipertensão, diabetes Mellitus; problemas: cardíacos, neurológicos, na coluna, nos joelhos, na tireoide; refluxo, hérnia de hiato, psoríase, ansiedade, enxaqueca, depressão, distúrbio do humor, problemas gástricos, lombalgia, renite, candidíase, varizes, asma, obesidade, glaucoma, endometriose. Dos 173 trabalhadores, 36\% fazem algum tipo de tratamento para as doenças relatadas.

A qualidade do sono é afetada pelo trabalho noturno, conforme a Tabela 2.

Tabela 2 - Frequência da qualidade do sono noturno após atividade laboral e dos sinais e sintomas relatados pelos trabalhadores após descanso noturno. Curitiba, 2008

\begin{tabular}{lc}
\hline Sono noturno & $N^{o}$ de Funcionários \\
\hline Tem dificuldade para dormir & 40 \\
Acorda várias vezes à noite & 74 \\
Dorme a noite toda sem interrupção & 74 \\
Outros & 7 \\
Acorda poucas vezes & 3 \\
Ao despertar se sente & \\
Descansado & 69 \\
Cansado & 93 \\
Outros & 7 \\
Lombalgia & 4 \\
Desânimo & 3 \\
Sono & 1 \\
Cefaleia & 2 \\
Queimação em MMII & 1 \\
Corpo pesado & 1 \\
Irritabilidade & 1 \\
Deprimido & 1 \\
Não responderam & 4 \\
\hline
\end{tabular}

* A porcentagem total ultrapassa o percentual máximo tendo em vista que muitos trabalhadores realizaram múltipla escolha

Os sintomas registrados após o descanso confirmam que o trabalhador ainda sente-se cansado, desanimado, bem como permanece ainda com sono.

\section{DISCUSSÃO}

Percebe-se que esses trabalhadores de enfermagem do período noturno estão em uma idade de plena capacidade de produção, fato encontrado em outros estudos $^{(3-11-12)}$, que reforçam o cuidado que devemos ter com o fenômeno "trabalhador sadio" em pesquisas, pois o trabalhador doente muitas vezes está em licença médica.

A predominância de mulheres na enfermagem é encontrada em outros estudos, mostrando a inserção das mulheres no mercado de trabalho para a contribuição da renda familiar e constitui um fator relevante para a sobrecarga de trabalho entre trabalhadoras mulheres. $\mathrm{O}$ trabalho remunerado é somado às atividades domésticas no período que seria de descanso, fator que contribui para um maior desgaste físico ${ }^{(3)}$.

O trabalho noturno para as mulheres pode também estar associado aos cuidados com os afazeres domésticos, como mostra esse estudo, na Tabela 1, 
bem como possuir outros vínculos empregatícios, o que compromete ainda mais o processo saúde-doença expresso pelos trabalhadores de enfermagem ${ }^{(14)}$. Dos trabalhadores entrevistados $74 \%$ possuem filhos, necessitando ajudar na renda familiar e contribuir na educação dos filhos. Outros estudos confirmam a necessidade do trabalhador possuir mais de um vínculo empregatício contribuindo na renda familiar ${ }^{(4,6-9)}$.

Um estudo com trabalhadores hospitalares descreve que a maioria dos entrevistados relatou que trabalhar a noite é muito cansativo e estressante, potencializado pelo fato de exercerem outras atividades durante o $\operatorname{dia}^{(12)}$.

A necessidade de outro vínculo empregatício vem para suprir necessidades, auxiliar no sustento do lar, outras vezes, são as únicas provedoras do lar e buscam mais tempo para auxiliar na educação dos filhos e conciliar os estudos ${ }^{(9,12-16)}$. Às vezes isso ocorre por um determinado tempo, até poderem suprir algumas necessidades almejadas, que depois de conquistadas, os fazem sair do período noturno.

As morbidades e sintomas relatados mostram o quão desgastante são suas atividades e o quanto afeta suas condições de saúde. Os sintomas mais expressos são varizes, fadiga, cervicodorsolombalgia e irritabilidade, em um percentual acima de 50\%.

Estudos têm mostrado como podem ocorrer distúrbios osteo-musculares devido à diversidade de tarefas realizadas pela enfermagem, desde o preparo de medicação, a verificação dos sinais vitais até a mobilização do paciente de um lugar para o outro ou de mudá-lo de uma posição para a outra, pois quanto maior o grau de dependência do paciente, maior a carga de trabalho pelos cuidados a prestar, bem como a duração das posturas penosas ${ }^{(9)}$.

Sintomas como dor de cabeça, nervosismo, depressão, dores no corpo, tontura, aversão à claridade, aumento do tom de voz, diminuição da acuidade visual, taquicardia, aparecimento de rugas, diminuição do desejo sexual, excesso de peso devido a alimentação inadequada, dor no estômago, náuseas e constipação foram relatados por trabalhadores de enfermagem noturno ${ }^{(11-12)}$.

O cansaço mesmo após o período de sono, a irritabilidade mental, a disposição para depressão, a perda da vitalidade e o pouco interesse no trabalho, assim como a perda de apetite ou mesmo os problemas digestivos podem ser consequência da fadiga crônica ${ }^{(13)}$.

A fadiga é o funcionamento excessivo do organismo humano, sendo consequência da requisição intensa e prolongada de músculos, esqueleto, órgãos sensoriais como olho e ouvido, e do cérebro, quer ele em atividade intelectual ou no trabalho físico coordenando a atividade de outros órgãos.

A cronificação da fadiga deve-se a um quadro de fadiga acumulada devido a situação de trabalho que não permite suficiente recuperação, mesmo depois do sono e repouso ${ }^{(9)}$. A fadiga visual é conhecida como astenopia caracterizada por dor e ardor nos olhos, vermelhidão em conjuntiva, piscar frequente, lacrimejamento, intolerância à claridade (fotofobia), visão dupla (diplopia), sensação de visão velada, percepção de auras coloridas em torno dos objetos e instabilidade da imagem, seguidos de outros sinais e sintomas físicos como cefaleia que se agrava com o cumprimento da tarefa, vertigem, manifestações gastrointestinais, desconforto e irritabilidade fácil, que geralmente está relacionada com a má iluminação ${ }^{(14)}$.

Outros estudos corroboram a piora na qualidade de sono dos trabalhadores noturnos. Os trabalhadores que conseguem dormir pela manhã após atividade laborativa noturna, apresentam um sono com duração que varia de 2 a 4 horas $^{(11-17)}$, o que confirma que 0 trabalhador retorna ao trabalho em situação de fadiga, diminuindo o rendimento físico, o nível de atenção e perturbando sensivelmente a coordenação motora e o ritmo mental, por isso o sono e o descanso adquirem especial importância para o trabalhador noturno, confirmada pela literatura ${ }^{(11)}$.

\section{CONSIDERAÇÕES FINAIS}

Este estudo mostrou que o desgaste do trabalhador de enfermagem noturno é uma realidade neste hospital. As consequências acarretadas à sua saúde e bem-estar deve ser fonte de preocupação para gestores e dirigentes institucionais, implicando na busca de soluções. Faz-se necessária a ocorrência de mudanças nas esferas organizacional e social, no modo da sociedade valorizar o trabalho, de maneira a também valorizar e construir formas de organizar o trabalho para uma melhor qualidade de vida do trabalhador.

Em virtude da ocorrência de agravos à saúde dos profissionais do período noturno, também se faz necessário e importante o desenvolvimento de projeto e implementação de ações voltadas à promoção e prevenção da saúde desta população, bem como minimizar os agravos já existentes. Poder-se-ia, por exemplo, garantir horários de descanso com o oferecimento de locais confortáveis, com boa 
ventilação/refrigeração, lanches nutritivos, ambientes silenciosos, camas confortáveis. A manutenção de escalas noturnas completas, com trabalhadores que possam suprir as eventuais faltas, poderia diminuir os frequentes aumentos da carga de trabalho noturno, principalmente a carga fisiológica, responsável pelos adoecimentos próprios da fadiga crônica.

Estudos futuros devem ser desenvolvidos no sentido de testar essas estratégias na melhoria das condições de trabalho e da qualidade de vida destes trabalhadores, e contribuir para reduzir os processos de morbidade que esse trabalho envolve.

\section{REFERÊNCIAS}

1. Brasil. Política Nacional de Segurança e Saúde do Trabalhador. Portaria Interministerial n ${ }^{\circ} 800$ de 03/05/ 05. Disponível: http://www.saude.gov.br

2. Mendes R. Patologia do trabalho. São Paulo: Atheneu; 2005.

3. Sarquis LMM. O monitoramento do trabalhador de saúde, após exposição a fluídos biológicos [tese]. São Paulo (SP): Escola de Enfermagem, Universidade de São Paulo; 2007.

4. Oliveira MM de. Alterações psicofisiológicas dos trabalhadores de enfermagem no serviço noturno [dissertação]. Programa de Pós-graduação em Enfermagem, Escola de Enfermagem Anna Nery, da Universidade Federal do Rio de Janeiro [série online]. Rio de Janeiro; 2005. [acesso em 2007 Out 03]. Disponível:http://www.scielo.br/scielo.php?script=sci_ arttext\&pid=S0104-07072009000200013\&lng=e\&nrm=is o\&tlng $=\mathrm{e}$

5. Marques N, Menna-Barreto L, organizadores. Cronobiologia: princípios e aplicações. $3^{\mathrm{a}}$ ed. São Paulo: Universidade de São Paulo; 2003.

6. Pires MLN, Squarcini CFR, Bittencourt LRA, Silva RS, Mello MT, Tufik S. Os ritmos circadianos e os comportamentais: alguns aspectos relevantes no trabalho por turnos e no noturno. In: Mello MT, Bittencourt LRA, Pires MLN, Silva RS, Tufik S, editores. Sono: aspectos profissionais e suas interfaces na saúde. São Paulo: Atheneu; 2008. p. 15-26.

7. Regis Filho GI, Sell I. Síndrome da má-adaptação ao trabalho em turnos: uma abordagem ergonômica. Itajaí: UNIVALI; 2000.

8. Campos MLP, De Martino MMF. Aspectos cronobiológicos do ciclo vigília-sono e níveis de ansiedade dos enfermeiros nos diferentes turnos de trabalho [periódico na Internet]. Rev Esc de Enferm USP.
2004 [acesso em 2008 Jun 08]; 38(4). Disponível: http:/ /www.scielo.br/scielo.php?pid=S0080-62342004000400 007\&script=sci_arttext

9. Bulhões I. Riscos dos trabalhos de enfermagem. $2^{\mathrm{a}}$ ed. Rio de Janeiro: Editorra?1998.

10. Ministério da Saúde (BR). Doenças relacionadas ao trabalho: manual de procedimentos para os serviços de saúde. Brasília; 2001.

11. Rezende MP. Agravos à saúde de auxiliares de enfermagem resultantes a exposição ocupacional aos riscos físicos [dissertação]. Escola de Enfermagem de Ribeirão Preto. Universidade de São Paulo [série online]. Ribeirão Preto; 2003 [acesso em 2008 Mai 03]. Disponível: http://www.cepis.ops-oms.org/bvsacd/ cd49/agravos.pdf

12. Furlani D. As necessidades humanas básicas de trabalhadores noturnos permanentes de um Hospital Geral frente ao não atendimento da necessidade sono [dissertação]. Curso de Pós Graduação em Engenharia da Produção. Universidade Federal de Santa Catarina [série online]. Florianópolis; 1999. [acesso em 2007 Out 20] Disponível: http:www.eps.ufsc.br/disserta99/ furlani/index/html

13. Kroemer KHE, Grandjean E. Manual de ergonomia. Adaptando o trabalho ao homem. $5^{\mathrm{a}}$ ed. Porto Alegre: Bookman; 2005.

14. Safiano CM, Sarquis LMM, Felli VEA, Giacomozzi LM. O processo saúde doença vivenciado pelos trabalhadores de enfermagem em uma instituição hospitalar [periódico na Internet]. Cogitare Enferm. 2003 [acesso em 2008 Maio 10]; v.8,n.2. Disponível: htpp:// ojs.c3sl.ufpr.br/ojs2/index.php/cogitare/article/ viewFile/1698/1406

15. Mello MT, Bittencourt LRA, Pires MLN, Silva RS, Tufik S. Sono: Aspectos profissionais e suas interfaces na saúde. São Paulo: Atheneu; 2008.

16. Magalhães AMM, Martins CMS, Falk MLR, Fortes CV, Nunes VB. Perfil dos profissionais de enfermagem do turno noturno do Hospital de Clínicas de Porto Alegre [periódico na Internet]. Rev HCPA. 2007 [acesso em 2008 Abr 05]. Disponível: http://www6.ufrgs.br/seer/ojs/ index.php/hcpa/article/viewFile/2016/1114

17. Borges FNS. Trabalhadores de enfermagem: compreendendo condições de vida e trabalho e ritmos biológicos [tese]. Faculdade de Saúde Pública. Universidade de São Paulo [série online]. São Paulo; 2006 [acesso em 2008 Mai 10]. Disponível: http:// www.teses.usp.br/teses/disponiveis/6/6134/tde07032007-1550549/ 